\title{
Carvedilol-B-cyclodextrin Systems: Preparation, Characterization and in vitro Evaluation
}

\author{
Anshu Sharma ${ }^{1}$ and C.P. Jain ${ }^{2}$ \\ ${ }^{1}$ Bhupal Nobles' College of Pharmacy, Udaipur - 313 001, Rajasthan, India. \\ ${ }^{2}$ Department of Pharmaceutical Sciences, MohanLal Sukhadia University, Udaipur-313 001, Rajasthan, India.
}

\begin{abstract}
The purpose of this study was to improve the solubility and dissolution rate of carvedilol by forming a complex with $\beta$-cyclodextrin. Phase solubility diagrams revealed increase in solubility of the drug upon cyclodextrin addition, showing $A_{N}$ type curve. Complexation of carvedilol was carried out with $\beta$-cyclodextrin by physical mixing, kneading and co-precipitation method. The prepared complexes and physical mixture were characterized by Fourier transform infra red spectroscopy, differential scanning calorimetry, powder X-ray diffractometry and inclusion efficiency. It was also observed that the complexes exhibit higher dissolution rates than the pure drug and physical mixture. Among all carvedilol-cyclodextrin complexes, inclusion complex (1:5) prepared by co-precipitation method showed better release.
\end{abstract}

Key words: Carvedilol, $\beta$-cyclodextrin, dissolution; solubility; stability study

\section{INTRODUCTION}

Cyclodextrins (CDs) have been developing increasing interest in the pharmaceutical field because of their ability to modify physical, chemical and biological properties of a number of hydrophobic drug molecules through the formation of inclusion complexes. ${ }^{1-3}$ They have been widely used as complexing agents to modify drug solubility or improve drug stability, and bioavailability, by means of drug inclusion into the hydrophobic cavity of cyclodextrin. CDs are thus offering new hope to formulation scientists in their efforts to develop an effective drug delivery system. CDs are effectively used as drug carriers and in foods and flavors, cosmetics, packing materials, textiles, separation processes, environmental protection efforts, fermentation, and catalysis. ${ }^{4-5}$

CDs are cyclic oligosaccharides consisting of $(\alpha-1,4)$-linked $\alpha$-D-glucopyranose units, with a relatively hydrophobic central cavity and a hydrophilic outer surface. The most abundant natural

Correspondence to: Anshu Sharma

E-mail: anshukiransharma@gmail.com

Dhaka Univ. J. Pharm. Sci. 12(1): 51-58, 2013 (June)
CDs are $\alpha$-cyclodextrin $(\alpha-C D), \quad \beta$-cyclodextrin $(\beta-\mathrm{CD})$, and $\gamma$-cyclodextrin $(\gamma-\mathrm{CD})$, containing 6,7 , and 8 glucopyranose units, respectively. The hydrophilic exterior surface of the $\mathrm{CD}$ molecules makes them water-soluble, but the hydrophobic cavity provides a microenvironment for appropriately sized nonpolar molecules. CDs are capable of forming inclusion complexes with many drugs by including a whole drug molecule, or only some nonpolar part of it, inside their cavity. In an aqueous solution, the complexes are readily dissociated and free drug molecules are in relatively rapid dynamic equilibrium with drug molecules bound within the CD cavity. ${ }^{6-9}$

Carvedilol competitively blocks $\beta 1, \beta 2$ and $\alpha 1$ receptors. It lacks sympathomimetic activity and has vasodilating properties that are exerted primarily through blockade. Carvedilol is used in the management of hypertension and angina pectoris, and as an adjunct to standard therapy in symptomatic heart failure. Carvedilol belongs to class II of the Biopharmaceutical classification systems, as it demonstrates poor solubility and high permeability. ${ }^{10-11}$ The major drawback with 
carvedilol therapy is its poor aqueous solubility and dissolution in gastric fluid. Hence it is aimed to enhance the aqueous solubility and dissolution rate of carvedilol by forming an inclusion complex with $\beta$-CD.

In the present study carvedilol and $\beta$-CD binary systems were prepared according to different techniques (physical mixing, kneading and co-precipitation method) and characterized by differential scanning calorimetry (DSC), powder X-ray diffractometry (PXRD), and Fourier transform infrared (FTIR) spectroscopy in order to achieve an improvement on carvedilol dissolution properties useful for different applications.

\section{MATERIALS AND METHODS}

\section{Materials}

Carvedilol was provided by Sun Pharmaceutical Ltd., Baroda, India as a gift sample and $\beta-C D$ was purchased from Hi-Media Laboratories Pvt. Ltd., Mumbai, India. All other chemicals and reagents used were of analytical grade.

\section{Methods}

Preparation of physical mixture by trituration method. Carvedilol and $\beta-C D$ in the ratio of $1: 1,1: 3$, 1:5 were sifted through a 40 -mesh $(425 \mu \mathrm{m})$ screen, mixed together (with trituration in a pestle mortar), and stored in a desiccated environment. ${ }^{12}$

Preparation of complexes by coprecipitation method. Carvedilol and $\beta-\mathrm{CD}$ were taken in ratio of $1: 1,1: 3$ and 1:5. The $\beta-C D$ was dissolved in adequate volume of methanol. The solvent was then rapidly evaporated with the aid of mild heat (up to about $50^{\circ} \mathrm{C}$ ) and surface airflow with constant vigorous stirring to form a uniform solid mass. The coprecipitate was crushed and desiccated under vacuum for $24 \mathrm{~h}$, pulverized (again, after formation of a more fragile mass), vacuum desiccated again for a day, sized into different sieve fractions and stored in a desiccators, until further use. ${ }^{13}$

Preparation of complexes by kneading method. Kneaded complexes were prepared by wetting physical mixture in different ratio of 1:1, 1:3 and 1:5 in mortar in least amount of methanol-water mixture (in $1 \% \mathrm{v} / \mathrm{v}$ ) and kneading thoroughly with a pestle to obtain a homogenous paste which was then dried in oven at $40^{\circ} \mathrm{C}$ for 48 hours. The dried complexes were pulverized into a fine powder and stored in a dessicator until further evaluation. ${ }^{14}$

Phase solubility studies. The phase solubility studies of carvedilol at various concentration of $\beta-C D$ was studied by the method reported by Higuchi and Connors. ${ }^{15}$ An excess amount of the drug was added to $10 \mathrm{ml}$ volumetric flask containing increasing concentration of $\beta-\mathrm{CD}$, in the range of $2 \mathrm{mM}$ to $14 \mathrm{mM}$ aqueous solution. The samples were allowed to shake for $48 \mathrm{~h}$ at $25 \pm 1^{\circ} \mathrm{C}$, until the equilibrium was established. The solutions were filtered through membrane filter $(0.45 \mu)$. After $48 \mathrm{~h}$, the carvedilol concentration was determined spectrophotometrically at $288 \mathrm{~nm}$ using Shimadzu UV 1800, Japan. The apparent 1:1 stability constants, $\mathrm{Kc}_{1: 1}$, were calculated from the linear portion of phase solubility diagrams using the equation:

$$
\mathrm{Kc}_{1: 1}=\text { slope } / \mathrm{S}_{0}(1 \text {-slope })
$$

where $S_{0}$ is the drug solubility in the absence of $\beta-C D$ (intercept).

Inclusion efficiency study. All inclusion complexes of carvedilol and their physical mixtures (25 mg) were separately taken in $25-\mathrm{ml}$ volumetric flasks. Ten milliliters of methanol were added to it, mixed thoroughly, and sonicated for $30 \mathrm{~min}$ at ambient temperature. The volume was made up to mark with methanol. An aliquot from each of the solution was suitably diluted with methanol to get the final concentration of $10 \mu \mathrm{g} / \mathrm{ml}$ of drug and spectrophotometrically assayed for drug content. Inclusion efficiency was calculated using the formula. ${ }^{16}$

Inclusion efficiency $=$ (estimated $\%$ drug content/ theoretical \% drug content) x 100

Fourier transform infrared spectroscopy. Fourier transform infrared spectra of carvedilol, $\beta-\mathrm{CD}$, physical mixtures and complexes were obtained using Shimadzu FTIR-8400S spectrometer, Japan. Samples of carvedilol, $\beta-C D$, physical mixtures and complexes were ground and mixed 
thoroughly with potassium bromide at a 1:5 sample/KBr ratio. The $\mathrm{KBr}$ discs were prepared by compressing the powders at a pressure of $5 \mathrm{~T}$ for 5 $\min$ in a hydraulic press. The scanning range was 40 to $4000 \mathrm{~cm}^{-1}$ and the resolution was $4 \mathrm{~cm}^{-1}$.

Differential scanning calorimetry (DSC). Thermal characteristics of the complexes were determined using a DSC analysis of the samples, carried out on a Perkin-Elmer DSC7, USA. Samples (6.5-10 mg) were accurately weighed and heated on a closed aluminum pan at a heating rate of $10^{\circ} \mathrm{C} / \mathrm{min}$ over the temperature range of 5 and $300^{\circ} \mathrm{C}$. DSC analysis was carried out under nitrogen gas flow of $20 \mathrm{lb} / \mathrm{in}^{2}$.

Powder X-ray diffraction (PXRD). PXRD patterns were recorded using Philips PW 1729 X- ray generator, USA fitted with a copper target, a voltage of $40 \mathrm{kV}$, and a current of $30 \mathrm{~mA}$. The scanning rate was $1 \%$ min over a $2 \theta$ range of $1-50^{\circ}$. PXRD patterns were traced for pure drug, $\mathrm{CD}$, physical mixture, solid dispersions and $\mathrm{CD}$ complexes. The samples were slightly ground and packed into the aluminum sample container.

In vitro studies. The release rate of carvedilol from physical mixtures and complexes was determined using United States Pharmacopoeia (USP) Dissolution Testing Apparatus 2 (paddle method; Veego Scientific, Mumbai, India). The dissolution test was performed using simulated gastric fluid for carvedilol at $37 \pm 0.5^{\circ} \mathrm{C}$ and $50 \mathrm{rpm}$ for 90 minutes. A $5 \mathrm{ml}$ aliquot was withdrawn at different time intervals and filtered using a $0.45 \mu$ nylon disc filter; each sample was replaced with $5 \mathrm{ml}$ of fresh dissolution medium. The filtered samples were suitably diluted, if necessary and assayed by measuring the absorbance spectrophotometrically at $288 \mathrm{~nm}$.

\section{RESULT AND DISCUSSION}

Phase solubility studies. The phase-solubility diagram for complex formation between carvedilol and $\beta-\mathrm{CD}$ is presented in Figure 1. According to the Higuchi and Connors classification, the diagram shows the $A_{N}$ type curve where the solubility increases linearly with $\mathrm{CD}$ concentration and further deviates negatively at higher concentrations. The interaction mechanism for the $A_{N}$ type curve is complicated because of a significant contribution of solute-solvent interaction to the complexation. The negative deviation from linearity may be associated with $\mathrm{CD}$ induced changes in the dielectric constant of the aqueous complexation media, changes in complex solubility and/or self-association of CD molecules. It can be seen from the curve that the apparent solubility of carvedilol increased linearly due to the formation of a soluble inclusion complex between carvedilol and $\beta-\mathrm{CD}$.

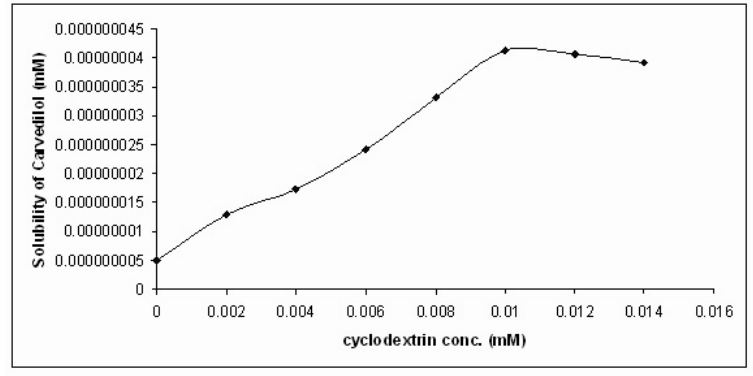

Figure 1. Phase solubility studies of Carvedilol.

In the region where a linear increase was observed, a linear regression analysis was performed and the stoichiometry of the complexes was assumed to be 1:1. The apparent association constant $\left(\mathrm{Kc}_{1: 1}\right)$ often plays an important role in explaining the various results obtained. The $\mathrm{Kc}$ for the complex formed was calculated from the slope (Higuchi and Connors, 1965).

The value of the stability constant $\mathrm{Kc}_{1: 1}$ was 610 $\mathrm{M}^{-1}$ for carvedilol, well within the range of 100 to $1000 \mathrm{M}^{-1}$ considered as ideal. A smaller $\mathrm{Kc}_{1: 1}$ value indicates too weak an interaction, whereas a larger value indicates the possibility of limited release of drug from the complex thereby interfering with drug absorption.

Inclusion efficiency study. The inclusion efficiency of the drug in $\beta$-CD was determined based on the differential solubility of free drug and complexed drug. The results of inclusion efficiency study are shown in Table 1 . The data indicate that the percent inclusion efficiency of 1:5 inclusion 
complexes prepared by co-precipitation method was $98.6 \% \pm 1.5$, whereas other inclusion complexes prepared by kneading method and physical mixtures have values in the range of $53.5 \pm 1.5$ to $84.6 \pm 1.53$ suggesting that drug was uniformly distributed in all 1:5 inclusion complex whereas the inclusion complexes and physical mixtures prepared in other ratios did not show satisfactory drug incorporation. Physical mixture and complex prepared by kneading and coprecipitation method having ratio 1:5 were further selected for FTIR, DSC, PXRD, in-vitro and stability studies.

Table 1. Inclusion efficiency study of carvedilol complexes and physical mixtures with $\beta$-cyclodextrin.

\begin{tabular}{lll}
\hline Methods & Ratio & $\begin{array}{l}\text { \% Inclusion } \\
\text { efficiency } \pm \text { SD }\end{array}$ \\
\hline Co-precipitation & $1: 1$ & $76.3 \pm 2.12$ \\
method & $1: 3$ & $86.2 \pm 2.23$ \\
& $1: 5$ & $98.6 \pm 1.50$ \\
Kneading & $1: 1$ & $65.36 \pm 2.31$ \\
method & $1: 3$ & $77.96 \pm 1.87$ \\
& $1: 5$ & $84.6 \pm 1.53$ \\
Physical & $1: 1$ & $53.5 \pm 1.50$ \\
Mixture & $1: 3$ & $62.6 \pm 0.81$ \\
& $1: 5$ & $67.5 \pm 1.2$ \\
\hline
\end{tabular}

Fourier transform infrared spectroscopy. The FTIR analysis is a useful technique to assess the interaction and the complex formation between drug molecules and CD in the solid state. This allows the detection of complex formations in solid phase and to point out the implication of the different functional groups of the guest and host molecules in the inclusion process, by analysing the significant changes in the shape and position of the absorbance bands. Shifts or intensity changes in the characteristic bands of pure substance are considered as evidence of the complex existence. ${ }^{17}$

Carvedilol showed characteristic peaks at $3346.27 \mathrm{~cm}^{-1}(\mathrm{O}-\mathrm{H}$ and $\mathrm{N}-\mathrm{H}$ stretching vibration peaks merged together), $2925.81 \mathrm{~cm}^{-1} \quad(\mathrm{C}-\mathrm{H}$ stretching vibrations), $1598.88 \mathrm{~cm}^{-1}(\mathrm{~N}-\mathrm{H}$ bending vibrations) and $1253.64 \mathrm{~cm}^{-1}$ (O-H bending and $\mathrm{C}-\mathrm{O}$ stretching vibrations). The spectrum of $\beta-\mathrm{CD}$ displayed a broad band between $3100 \mathrm{~cm}^{-1}$ and 3800 $\mathrm{cm}^{-1}$ attributed to free $\mathrm{OH}$ from primary and secondary $\mathrm{OH}$ groups, between $2800 \mathrm{~cm}^{-1}$ and 3100 $\mathrm{cm}^{-1}$ corresponding to bound $\mathrm{OH}$, at $1626 \mathrm{~cm}^{-1}$ due to water molecules present in the cavity, and a large band that displayed distinct peaks between $900 \mathrm{~cm}^{-1}$ and $1200 \mathrm{~cm}^{-1}$, responsible for C-O vibrations. The FTIR spectra (Figure 2) of the inclusion complex prepared by co-precipitation, kneading method and

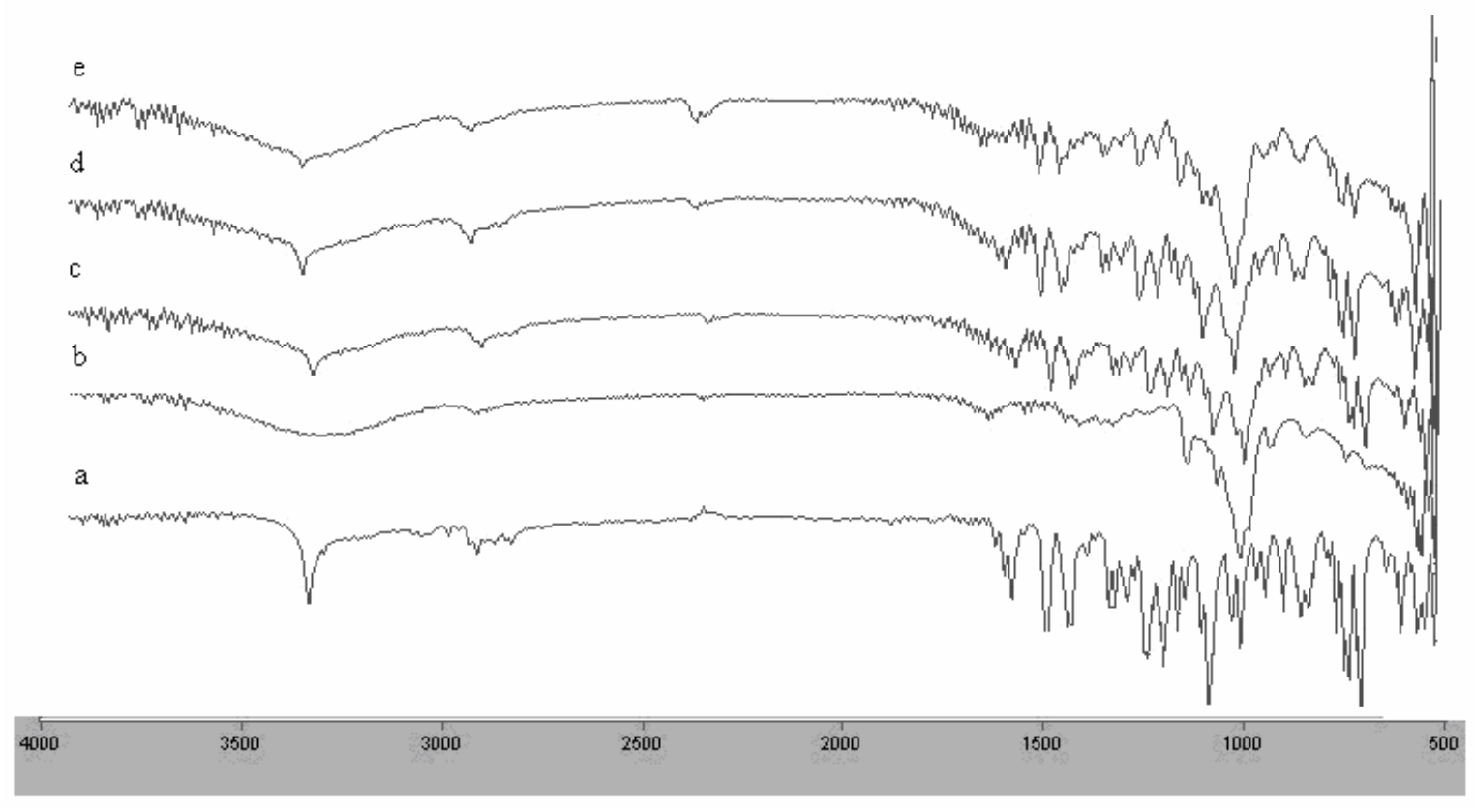

Figure 2. FTIR spectra of a) carvedilol b) $\beta$-cyclodextrin c) physical mixture d) kneaded complex e) co-precipitated complex. 
physical mixture were dominated by the vibrational bands of the CD molecule. Any sign of interaction on complexation would be reflected by changes in the characteristic peaks of carvedilol, depending on the extent of interaction. The FTIR spectra of all the complexes did not show significant differences from the respective spectra of carvedilol and $\beta-\mathrm{CD}$ except shift of peak at $1253.64 \mathrm{~cm}^{-1}(\mathrm{O}-\mathrm{H}$ bending and $\mathrm{C}-\mathrm{O}$ stretching vibrations) to $1252.57 \mathrm{~cm}^{-1}$ and 1252.12 $\mathrm{cm}^{-1}$ for the complexes prepared by kneading and co-precipitation method, respectively which is due to the inclusion complexes. The physical mixture exhibited a peak indicating alkyl aryl stretching at $1212.60 \mathrm{~cm}^{-1}$ and $1020 \mathrm{~cm}^{-1}$ indicating a weak interaction between carvedilol and $\beta-\mathrm{CD}$.

Differential scanning calorimetry (DSC). DSC is a useful technique for drugs that form the inclusion complexes with $\beta$-CD. The disappearance or reduction of the endotherm related to the melting of the crystalline drug in the DSC profile of the drug/ $\beta$ $\mathrm{CD}$ complex is generally taken as an indication of the interaction between the components.
The DSC curve of carvedilol showed a sharp endothermic peak (Tpeak $=115^{\circ} \mathrm{C}$ ) corresponding to its melting point, indicating its crystalline nature. $\beta$-CD exhibits a broad endothermic effect ranging between $30^{\circ} \mathrm{C}$ and $149^{\circ} \mathrm{C}$ associated with loss of water from inside the cavity. The thermal behavior (Figure 3) of both physical mixture and complexes of the drug was different. In case of physical mixture, the peak of carvedilol was weekend, broadened and appeared at $110^{\circ} \mathrm{C}$. These findings demonstrated the presence of interactions between the drug and $\mathrm{CD}$ system. In the complex prepared by kneading method, the peak of carvedilol appeared at $110^{\circ} \mathrm{C}$ but largely broadened and weakend. In the DSC curve of the complex prepared by co-precipitaion method, the sharp fusion peak of carvedilol was not observed, indicating the interaction the carvedilol with $\mathrm{CD}$ cavity, which in turn leads to an almost complete loss of crystallinity in the binary system. The differences in the thermal behavior of carvedilol in form of physical mixtures and complexes suggested the drug crystallinity decreased when prepared as complexes and that decrease was dependent on the method of preparation of complexes.

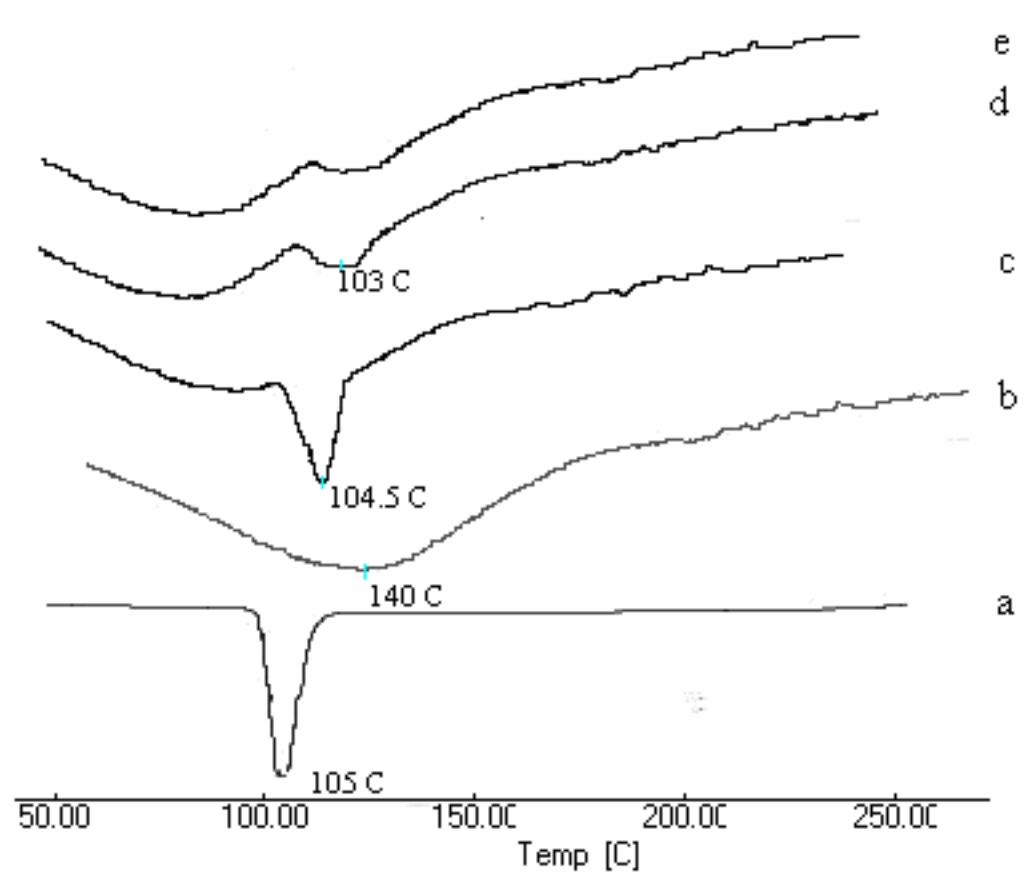

Figure 3. DSC spectra of a) carvedilol b) $\beta$-cyclodextrin c) physical mixture d) kneaded complex e) co-precipitated complex. 
Powder X-ray diffraction (PXRD). Powder XRD study was used to measure the crystallinity of the formed inclusion complexes. A change in the crystallinity of the drug indicates complex formation by appearance of a new or at least deviation from the original pattern. The formation of an amorphous state proves that the drug was dispersed in a molecular state with CDs.

Numerous diffraction peaks of carvedilol were observed at $2 \theta$ of $12.8^{\circ}, 15.62^{\circ}, 17.46^{\circ}, 18.56^{\circ}$, $20.1^{\circ}, 24.3^{\circ}$ and $26.2^{\circ}$ indicating the presence of crystalline nature of carvedilol. $\beta-C D$ is a very crystalline molecule with major peaks at $2 \theta$ values of $4.75^{\circ}, 12.7^{\circ}, 19.7^{\circ}, 21.1^{\circ}, 22.8^{\circ}, 24.3^{\circ}$, and $35.9^{\circ}$.
XRD-scanning of physical mixture showed decreasing number of peaks with lower intensity indicating partial amorphous nature of the drug in its binary mixtures (Figure 4). In case of inclusion complex prepared by kneading method, there was a decrease in the intensity of carvedilol but the major peaks remained at the same positions. On the other hand, no diffraction peak from carvedilol was observed in complex prepared by coprecpitation method. The PXRD pattern of this complex was diffused. This result suggests that carvedilol exists in an amorphous state in the complex.

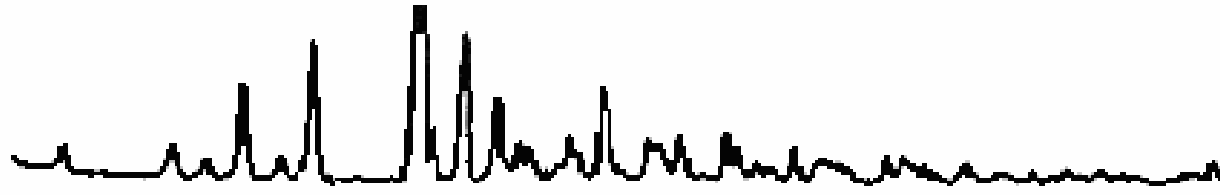

C

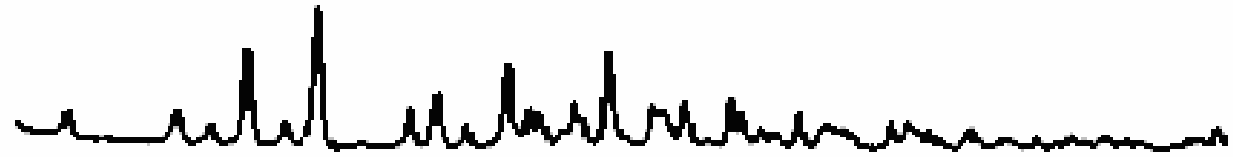

b

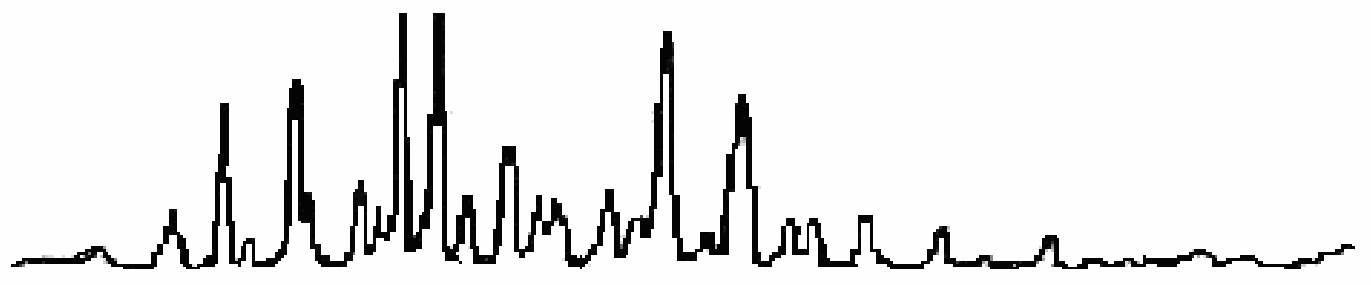

a
20
30

40 e

d

20 (degree)

Figure 4. PXRD diffractogram of a) carvedilol b) $\beta$-cyclodextrin c) physical mixture d) kneaded complex e) co-precipitated complex. 


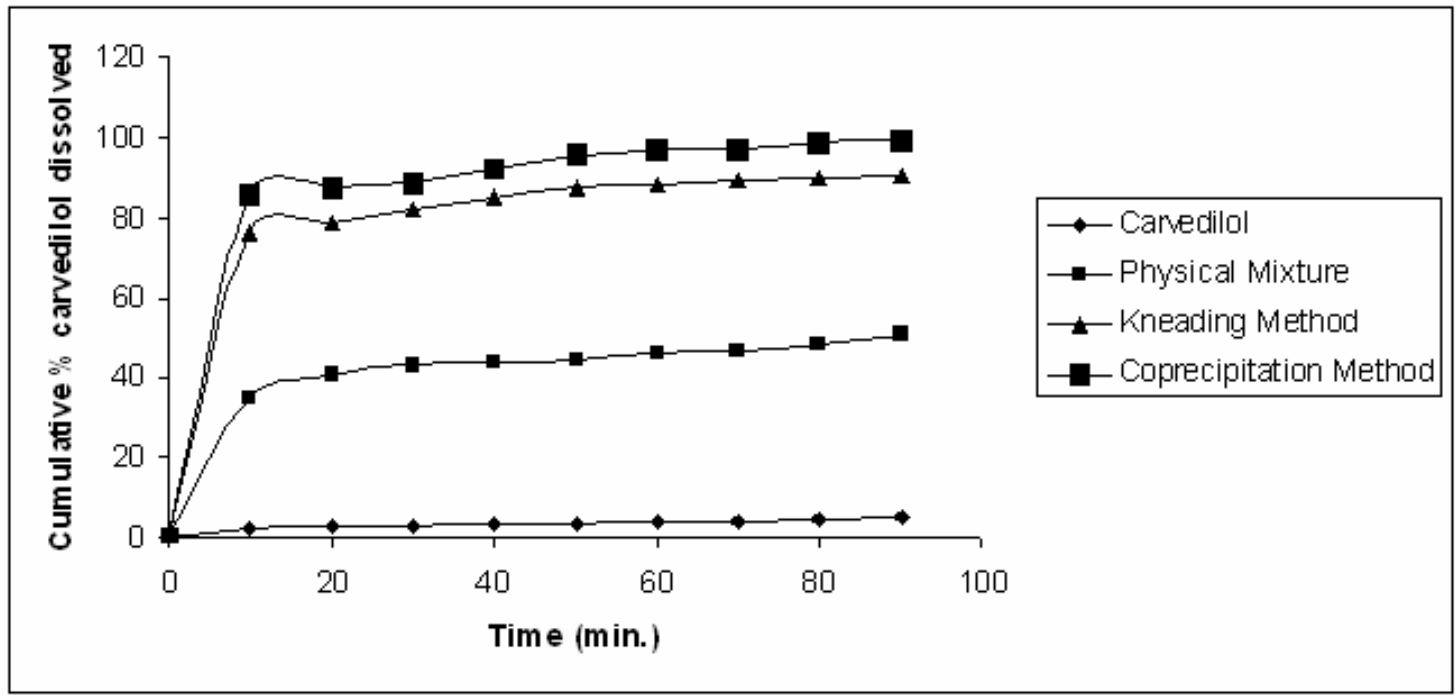

Figure 5. Dissolution profile of physical mixtures and complexes of $\beta$-cyclodextrin with Carvedilol.

In vitro studies. The dissolution profile of pure carvedilol and $\beta-\mathrm{CD}$ complexes formed by physical mixture, kneading method and co-precipitation methods are illustatrated in Figure 5. The dissolution profiles of all component exhibited first order release kinetics. All $\beta$-CD complexes exhibited higher rates of dissolution and dissolution efficiency values than pure drug, indicating rapid and higher dissolution of drug from its $\beta$-CD complexes. The drug release in $20 \mathrm{~min}$ for carvedilol (5.12\%) was enhanced in physical mixture (50.65\%), kneaded complex $(90.34 \%)$ as well as in co-precipitated complex $(99.36 \%)$.

Improved dissolution of the complexes was simultaneous with increased particle wettability and reduced crystallinity of the product. The surfactantlike properties of $\beta-\mathrm{CD}$ were postulated in some cases to explain the higher dissolution rate of the complexes. $\beta-\mathrm{CD}$ can reduce the interfacial tension between the solid particles of pure drug. The improvement in dissolution rate of pure drug from $\beta-C D$ complexes was in agreement with the results obtained from phase solubility analysis.

The extent of the enhancement of dissolution rate was found to depend on the preparation method of the complex. The improved dissolution of the complex prepared through kneading and coprecipitation may be due to the formation of an inclusion complex of the drug with $\beta$-CD and /or conversion of the drug to an amorphous state or nearly amorphous state supported by XRD and DSC studies. Physical mixtures demonstrated better dissolution rates because $\beta-C D$ dissolves more rapidly in the dissolution medium than the pure drug. It can be assumed that, in the early stages of dissolution process, the $\beta-C D$ molecules operate locally on the hydrodynamic layer surrounding the drug particles. This action results in an in situ inclusion process, which produces a rapid increase in the amount of dissolved drug.

\section{CONCLUSION}

The results suggest that $\beta-\mathrm{CD}$ forms inclusion complexes with carvedilol by both the kneading and co-precipitation techniques, with a dramatic improvement in drug dissolution rate relative to the drug or the $\beta-C D /$ drug physical mixture. The release profile of inclusion complexes prepared by coprecipitation techniques was better compared to that of complexes prepared by kneading method.

\section{REFERENCES}

1. Jug, M. and Becirevic-Lacan, M. 2004. Multicomponent complexes of piroxicam with cyclodextrins and hydroxypropyl methylcellulose. Drug Dev. Ind. Pharm. 30, 1051-1060 
2. Uekama, K., Hirayama, F. and Irie, T. 1998. Cyclodextrin drug carriers systems. Chem. Rev. 98, 2045-2076.

3. Manca, M.L., Zaru, M., Ennas, M., Valenti, D. Sinico, C. Loy, G. and Fadda, A.M. 2005. Diclofenac- $\beta$-cyclodextrin binary systems: physicochemical characterization and in vitro dissolution and diffusion studies. AAPS Pharm. Sci. Tech. 6, E464-E472.

4. Thompson, D.O. 1997. Cyclodextrins-enabling excipients: their present and future use in pharmaceuticals. Crit. Rev. Ther. Drug Carrier. Syst. 14, 1-104.

5. Masson, M., Loftsson, T., Masson, G. and Stefansson, E.S. 1999. Cyclodextrins as permeation enhancer some theoretical evaluations and in vitro testing. J. Control. Release. 59, 107118.

6. Chowdary, K.P.R. and Srinivas, S.V. 2006. Influence of hydrophilic polymers on celecoxib complexation with hydroxypropyl $\beta$-cyclodextrin. AAPS Pharm. Sci. Tech. 7, E1- E6.

7. Loftsson, T. and Brewster, M.E. 1996. Pharmaceutical applications of cyclodextrins, I: drug solubilization and stabilization. J. Pharm. Sci. 85, 1017-1025.

8. Rajewski, R.A. and Stella, V.J. 1996. Pharmaceutical applications of cyclodextrins, II: in vivo drug delivery. $J$. Pharm. Sci. 85, 1142-1169.

9. Larsen, K.L. 2002. Large cyclodextrins. J. Inclusion Phenom. Macro. Chem. 43, 1-13.

10. Ruffolo Jr., R.R., Boyle, D.A., Venuti, R.P. and Lukas, M.A. 1993. Preclinical and clinical pharmacology of carvedilol. $J$. Hum. Hypertens. 7, S2-S15.
11. Ruffolo Jr., R.R., Gellai, M., Hieble, J.P. Willette, R.N. and Nichols, A.J. 1990. The pharmacology of carvedilol. Eur. J. Clin. Pharmacol. 38, S82-S88.

12. Baboota, S., Dhaliwal, M. and Kohli, K. 2005. Physicochemical Characterization, In vitro dissolution behavior, and pharmacodynamic studies of rofecoxibcyclodextrin inclusion compounds. Preparation and properties of rofecoxib hydroxypropyl $\beta$-cyclodextrin inclusion complex: a technical note. AAPS Pharm. Sci. Tech. 6, E83-E90.

13. Longxiao, V. and Xiaocui, W. 2007. Improved dissolution of oleanolic acid with ternary solid dispersions. AAPS Pharm. Sci. Tech. 8, E1-E5.

14. Cunha-Filho, M.S.S., Dacunha-Marinho, B., TorresLabandeira, J.J., Martinez-Pacheco, R. and Landin, M. 2007. Characterization of $\beta$-lapachone and methylated $\beta$-cyclodextrin solid-state systems. AAPS Pharm. Sci. Tech. 8, E1-E10.

15. Higuchi, T. and Connors, K.A. 1965. Phase-solubility techniques. Adv. Anal. Chem. Instr. 14, 117-212, 1965.

16. Patel, S.G. and Rajput, S.J. 2009. Enhancement of oral bioavailability of cilostazol by forming its inclusion complexes. AAPS Pharm. Sci. Tech. 10, 660-669.

17. Cirri, M., Mura, P., Rabasco, A.M., Gines, J.M., Moyano, J.R. and Gonzalez-Rodriguez, M.L. 2004. Characterization of ibuproxam binary and ternary dispersions with hydrophilic carriers. Drug Dev. Ind. Pharm. 30, 65-74. 